\title{
Consistent High Resolution Interface-Capturing Finite Volume Method for Compressible Multi-material Flows
}

\author{
Qiuju Wang ${ }^{\mathrm{a}}$, Ralf Deiterding ${ }^{\mathrm{b}}$, Jianhua $\mathrm{Pan}^{\mathrm{c}}, \mathrm{Yu}-\mathrm{Xin} \mathrm{Ren}^{\mathrm{d}}$ \\ ${ }^{a}$ Institute of Applied Physics and Computational Mathematics, Beijing, 100094, China \\ ${ }^{b}$ University of Southampton, Southampton, SO171BJ, United Kingdom \\ ${ }^{c}$ Ohio State of University, Columbus, OH43210, USA \\ ${ }^{d}$ School of Aerospace, Tsinghua University, Beijing 100084, China
}

\begin{abstract}
Compressible multi-material flows are characterized by complex flow structures with a broad range of length scales and discontinuities associated with material interfaces and shock waves. High order and high resolution numerical methods are required to capture material interface as sharply as possible and to increase the resolution of complex structures along the material interface. This paper will present the application of the high resolution finite volume (FV) method based on the minimized dispersion and controllable dissipation (MDCD) reconstruction to compressible multi-material flows. The MDCD scheme with independent dispersion and dissipation provides a flexible mechanism to control the numerical dissipation. The adjustment of dissipation of the MDCD reconstruction will not affect the consistency, which is required for interface-diffusion capturing methods to prevent spurious oscillations. Several one- and two dimensional multi-material numerical simulations have indicated that the high resolution FV method based on MDCD reconstruction can capture material interfaces free of spurious oscillations and provide better resolved small-scale features than other numerical schemes on
\end{abstract}


the same grid resolution.

Keywords: Multimaterial, Interface-capturing, High-resolution, Finite volume method

\section{Introduction}

Compressible multi-material flows are usually associated with complex problems, such as inertial confinement fusion, high-speed impact, high-speed combustion, high-energy physics and supernovae explosions. The evolution of deformations near a material interface is important for compressible multimaterial problems. However, spurious oscillations are typically generated at material interfaces in simulations of compressible multi-material flows. Therefore, one challenge for simulations of compressible multi-material flows is how to handle material interfaces. There are generally two techniques used to capture material interfaces, which are sharp and diffusion interface methods [1]. In the first class, the interface is preserved as a resolved surface and each material aside from the interface is treated independently [2-10]. In the second class, the diffusion interface methods adopt a single set of equations to model the multi-material flows at all the computational cells and implicitly capture the interface separating different materials [11]. With this advantage, the diffusion-interface models [12-16] gain a great success for simulations of compressible multi-material flows. Another challenge is how to accurately simulate the compressible multi-material flows especially characterized by large interface deformations and mixing process with complex structures at small scales. Accordingly, high order and high resolution numerical methods are required to capture material interfaces as sharply as 
possible and to increase the resolution of small features along the interfaces for simulations of multi-material flows.

In this paper, we will propose a high-resolution FV method using MDCD reconstructions for solving compressible multi-material flows based on the single pressure and single velocity model. For single material flows, the MDCD technique proposed in the finite difference framework [17] has been extended to the FV method [18]. There are two advantages for the MDCD technique: (1) the dispersion error is minimized and one free parameter is left to control dissipation; (2) The optimized dispersion property is not affected by the dissipation adjustment. However, the MDCD technique developed for single material flows cannot be directly applied to compressible multimaterial flows. The challenges for high-resolution numerical algorithms of diffusion-interface methods are the consistency and the high resolution issues, which are crucial for the accurate treatment of material interfaces.

For the consistency issue, numerical algorithms are required to be consistent with both diffusion-interface models and interface conditions to prevent spurious oscillations. Diffusion-interface methods solve a material interface as a contact discontinuity in the diffused numerical zone which contains the artificial mixtures produced by numerical diffusion. Absent fulfillment of all the assumed interface conditions within the artificial mixtures, spurious velocity, pressure and temperature oscillations tend to appear in the diffusion zones and can propagate and contaminate the solution fields. The spurious oscillations are carefully analyzed and consistent physical models are proposed to prevent pressure oscillations and temperature spikes [19-27]. In this paper, we will apply high resolution numerical method to the governing 
model with physical and numerical consistency in mechanical and thermal equilibrium within artificial mixtures.

For the high-resolution treatment of material interfaces, some shock/interfacecapturing numerical methods tend to be too dissipative and numerically smear the material interface. Hence, the artificial diffusion introduced by interface-diffusion capturing methods should be alleviated as much as possible to sharpen the diffusion zones and to increase the resolution of complex structures along the material interface. Several numerical approaches have been developed to minimize the numerical smearing, which attempt to compress the interfaces [28-31], to sharpen the interfaces [32-34], as well as to adopt high-order numerical schemes [21, 26, 35-43]. Most recent developments tend to apply high-order algorithms to increase the resolution of the diffusive material interfaces. However, the order of the truncation error of a numerical scheme only provides information on the asymptotic convergence rate to the exact solution without enough information on the actual error on a finite computational grid [44]. Hence, spectral-like high resolution numerical methods are developed for simulations of flows with a broad range of length scales $[17,18,45-48]$. In this paper, we will apply high-resolution MDCD scheme for reconstructions of FV method to capture material interfaces.

There are several characteristics of the high resolution FV method with the MDCD reconstruction for compressible multi-material flows. Firstly, the MDCD reconstruction with minimized dispersion and controllable dissipation can more sharply capture material interfaces and improve the resolution of finer structures. Secondly, the MDCD reconstruction provides a very flexible mechanism to control the numerical dissipation of the numerical scheme. 
The adjustment of dissipation of the MDCD reconstruction will not affect the consistency, which is required for interface-diffusion capturing methods. Moreover, the adjustment of dissipation will not affect the optimized dispersion property. Thirdly, the nonlinear adaptation mechanism of the nonlinear MDCD scheme is optimized to reduce dissipations using the relative smoothness limiter [39]. Finally, the proposed FV method can naturally maintain discrete conservation of the total mass, momentum, energy and species mass for the compressible multi-material flows with material interfaces and discontinuities.

The remainder of this paper is organized as follows. In Section 2, the physical model is presented. Section 3 will illustrate the numerical methods. In Section 4, the numerical examples are provided to demonstrate the performance of the proposed scheme. Finally, the conclusion remarks are given in Section 5.

\section{Physical model}

\subsection{Governing equations}

The conservative equations of total mass, momentum and energy in the Euler system are given by

$$
\begin{aligned}
\frac{\partial \rho}{\partial t}+\frac{\partial \rho u_{j}}{\partial x_{j}} & =0 \\
\frac{\partial \rho u_{j}}{\partial t}+\frac{\partial}{\partial x_{k}}\left(\rho u_{j} u_{k}+p \delta_{j k}\right) & =0 \\
\frac{\partial E}{\partial t}+\frac{\partial}{\partial x_{j}}\left[u_{j}(E+p)\right] & =0
\end{aligned}
$$


where $\rho$ is density, $u_{j}$ is velocity, $p$ is pressure and $E$ is total energy. The total energy is

$$
E=\rho\left(e+\frac{u_{k} u_{k}}{2}\right)
$$

in which $e$ is internal energy.

For multi-fluid flows, Eqs.(1)-(3) are supplemented with $(N-1)$ additional conservative equations for species

$$
\frac{\partial\left(\rho Y_{i}\right)}{\partial t}+\frac{\partial\left(\rho Y_{i} u_{j}\right)}{\partial x_{j}}=0
$$

where $Y_{i}$ is mass fraction of species $i$ and $N$ is total number of the species.

\subsection{Mixing rules}

Mixing rules are used to calculate thermal dynamic relations for artificial mixtures in the diffusion zone. In this paper, the ideal gas law is adapted. Then the pressure is computed by

$$
\frac{p}{\gamma-1}=\rho e
$$

with the ratio of specific heats $\gamma$ derived by the following mixing rule

$$
\frac{1}{\gamma-1}=\sum_{i=1}^{N} \frac{Y_{i}}{\gamma_{i}-1} \frac{W}{W_{i}},
$$

where $W$ is the mixture molecular mass which is computed by

$$
\frac{1}{W}=\sum_{i=1}^{N} \frac{Y_{i}}{W_{i}}
$$

The temperature is computed by

$$
T=\frac{p}{\rho R}
$$

where $R$ is the mixture gas constant and is calculated by

$$
R=\sum_{i=1}^{N} Y_{i} R_{i}
$$




\subsection{Equilibriums}

\subsubsection{Equilibriums of velocity and pressure}

The transport equations in non-conservative form were proposed to prevent pressure oscillations along material interfaces [23-25]. In this paper, the transport equation in non-conservative form is adopted as follows:

$$
\frac{\partial \Gamma}{\partial t}+u_{j} \frac{\partial \Gamma}{\partial x_{j}}=0
$$

where $\Gamma=1 /(\gamma-1)$. Eqs.(1)-(4) and (10) form a quasi-conservative system [25]. The value of $1 /(\gamma-1)$ computed from Eq.(10) is only used to calculate pressure and is different from the value of that computed by mixing rules [39].

Besides the transport equation, the high-order and high resolution reconstructions of the primitive variables are required to maintain pressure equilibrium.

\subsubsection{Equilibriums of temperature}

High-order diffusion-interface methods generally result in temperature spikes and the temperature errors will become problematic when temperaturedependent problems are in considerations $[21,26]$. To prevent temperature spikes, $\rho$ and $\left(\rho Y_{i}\right)$ are required to use the same difference operations including reconstruction and upwinding. In this paper, the high resolution

reconstructions of $\rho$ and $\left(\rho Y_{i}\right)$ are conducted using the same weights for the nonlinear interpolations of $\rho$. 


\section{Numerical methods}

In the $\mathrm{FV}$ method, the primitive variables $\mathbf{U}=\left[\rho, \rho Y_{i}, u_{1}, u_{2}, u_{3}, p, \Gamma\right]^{\mathrm{T}}$ are required to be reconstructed to maintain velocity and pressure equilibrium. Moreover, $\rho$ and $\left(\rho Y_{i}\right)$ are reconstructed using the same weights to maintain temperature equilibrium. The Riemann problem from the reconstruction is solved by the HLLC Riemann solver $[35,36]$. The time marching is handled by third order total variation diminishing Runge-Kutta scheme (TVD-RK).

\subsection{Spatial discretization}

In the FV method formulation, the integral form of the quasi-conservative governing equations for compressible multi-material flows in Euler system is given by

$$
\frac{\partial}{\partial t} \int_{\Omega_{j}} \mathbf{Q} d \Omega+\int_{\partial \Omega_{j}} \mathbf{F} \cdot \mathbf{n} d S=\int_{\Omega_{j}} \boldsymbol{\omega} d \Omega .
$$

The spatially discretized finite volume scheme is

$$
\frac{\partial}{\partial t}\left(\overline{\mathbf{Q}}_{j} \Omega_{j}\right)+\sum_{f=1}^{m}(\mathbf{F} \cdot \mathbf{n} S)_{f}=\overline{\boldsymbol{\omega}}_{j} \Omega_{j}
$$

with $\mathbf{Q}=\left[\rho, \rho Y_{i}, \rho u_{1}, \rho u_{2}, \rho u_{3}, E, \Gamma\right]^{\mathrm{T}}$,

$$
(\mathbf{F} \cdot \mathbf{n} S)_{f}=\left[\begin{array}{l}
\rho \mathbf{u} \cdot \mathbf{n} S \\
\rho Y_{i} \mathbf{u} \cdot \mathbf{n} S \\
\left(\rho u_{1} \mathbf{u} \cdot \mathbf{n}+p n_{1}\right) S \\
\left(\rho u_{2} \mathbf{u} \cdot \mathbf{n}+p n_{2}\right) S \\
\left(\rho u_{3} \mathbf{u} \cdot \mathbf{n}+p n_{3}\right) S \\
(E+p) \mathbf{u} \cdot \mathbf{n} S \\
\Gamma \mathbf{u} \cdot \mathbf{n} S
\end{array}\right]_{f}, \overline{\boldsymbol{\omega}}_{j} \Omega_{j}=\left[\begin{array}{l}
0 \\
0 \\
0 \\
0 \\
0 \\
0 \\
\bar{\Gamma}_{j} \sum_{f=1}^{m}\left[(\mathbf{u} \cdot \mathbf{n})^{\mathrm{HLLC}} S_{f}\right]
\end{array}\right] .
$$


where $\mathbf{n}=n_{1} \mathbf{i}+n_{2} \mathbf{j}+n_{3} \mathbf{k}$ is the unit outward normal vector of a cell surface of the control volume and $\mathbf{u}=u_{1} \mathbf{i}+u_{2} \mathbf{j}+u_{3} \mathbf{k}$ is the velocity vector. $\overline{\mathbf{Q}}_{j}$ is the cell average value of $\mathbf{Q}$ in cell $I_{j}$. The flux at the cell surface $(\mathbf{F} \cdot \mathbf{n} S)_{f}$ is achieved by solving the Riemann problem with left and right states, which are reconstructed from cell averages. The velocity $(\mathbf{u} \cdot \mathbf{n})^{H L L C}$ in the source term of the transport equation is given in Section 3.3 with details.

\subsection{Reconstruction}

The high resolution MDCD scheme is used to reconstruct the left and right states of the Riemann problem. A fully downwind candidate stencil is added to the classical WENO stencils. Fig. 1 depicts the candidate stencils arrangements for $r=3$ which is symmetric about the cell interface $j+1 / 2$.

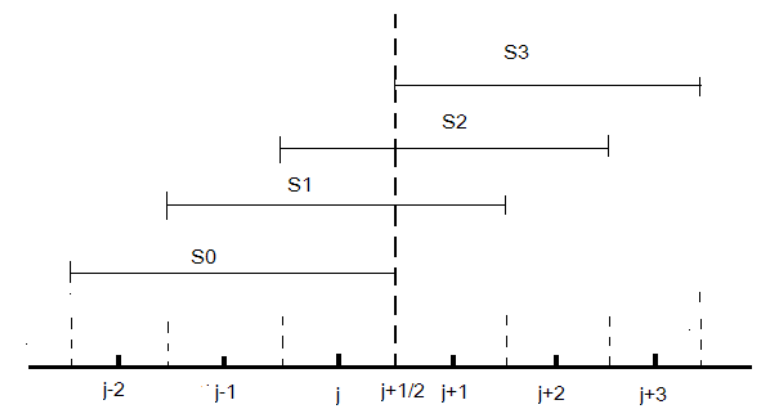

Figure 1: Symmetric stencil for $r=3$ with 6 candidates.

\subsubsection{The linear reconstruction}

In recognition that the optimal dispersion should be minimized according to some chosen criteria while the optimal dissipation is often problem dependent, the MDCD scheme $[17,18]$ is proposed by minimizing dispersion errors 
and leaving one free parameter for adjusting dissipations. The optimized dispersion properties are not affected by the dissipation adjustment.

For simplicity, we use the following one-dimensional case to illustrate the details of the reconstruction procedures. The cell averages $\bar{q}$ are used to reconstruct the left and right state at the cell interface. For the $r=3$ case, the left state $q_{j+\frac{1}{2}}^{L}$ and right state $q_{j+\frac{1}{2}}^{R}$ are computed by

$$
q_{j+\frac{1}{2}}^{L}=\sum_{k=0}^{r} C_{k} q_{j+\frac{1}{2}}^{k}, q_{j+\frac{1}{2}}^{R}=\sum_{k=0}^{r} C_{r-k} q_{j+\frac{1}{2}}^{k},
$$

where $q_{j+\frac{1}{2}}^{k}$ is obtained by a $2^{\text {nd }}$-degree polynomial reconstructed on stencil $S_{k}$ shown in Fig.1. The detailed formulation of $q_{j+\frac{1}{2}}^{k}$ is given by

$$
\begin{aligned}
q_{j+\frac{1}{2}}^{0} & =\frac{2}{6} \bar{q}_{j-2}-\frac{7}{6} \bar{q}_{j-1}+\frac{11}{6} \bar{q}_{j}, \\
q_{j+\frac{1}{2}}^{1} & =-\frac{1}{6} \bar{q}_{j-1}+\frac{5}{6} \bar{q}_{j}+\frac{2}{6} \bar{q}_{j+1}, \\
q_{j+\frac{1}{2}}^{2} & =\frac{2}{6} \bar{q}_{j}+\frac{5}{6} \bar{q}_{j+1}-\frac{1}{6} \bar{q}_{j+2}, \\
q_{j+\frac{1}{2}}^{3} & =\frac{11}{6} \bar{q}_{j+1}-\frac{7}{6} \bar{q}_{j+2}+\frac{2}{6} \bar{q}_{j+3},
\end{aligned}
$$

where $\bar{q}_{j}$ is the cell average

$$
\bar{q}_{j}=\frac{1}{h} \int_{x_{j-\frac{1}{2}}}^{x_{j+\frac{1}{2}}} q(x) d x .
$$

$C_{k}$ is the linear weight of the candidate stencil $S_{k}$ which is calculated by

$$
\begin{aligned}
C_{0} & =\frac{3}{2} \nu_{\text {disp }}+\frac{3}{2} \nu_{\text {diss }}, \\
C_{1} & =\frac{1}{2}\left(1-3 \nu_{\text {disp }}+9 \nu_{\text {diss }}\right), \\
C_{2} & =\frac{1}{2}\left(1-3 \nu_{\text {disp }}-9 \nu_{\text {diss }}\right), \\
C_{3} & =\frac{3}{2} \nu_{\text {disp }}-\frac{3}{2} \nu_{\text {diss }},
\end{aligned}
$$


where $\nu_{\text {disp }}$ is the dispersion parameter which is optimized by minimizing dispersion error and $\nu_{\text {diss }}$ is the dissipation parameter which is often problem dependent $[17,18]$. Therefore, in this paper, $\nu_{\text {disp }}$ and $\nu_{\text {diss }}$ are given as

$$
\nu_{d i s p}=0.0463783, \nu_{\text {diss }} \in[0,0.0463783] .
$$

This linear reconstruction will be called the (linear) MDCD scheme.

\subsubsection{The nonlinear reconstruction}

The linear reconstruction cannot directly be used to compute the flow fields with discontinuities. Therefore, the nonlinear adaptation mechanism of WENO is introduced to capture discontinuities.

The left state $q_{j+\frac{1}{2}}^{L}$ and right state $q_{j+\frac{1}{2}}^{R}$ are computed by nonlinear reconstruction

$$
q_{j+\frac{1}{2}}^{L}=\sum_{k=0}^{r} \omega_{k} q_{j+\frac{1}{2}}^{k}, q_{j+\frac{1}{2}}^{R}=\sum_{k=0}^{r} \omega_{r-k} q_{j+\frac{1}{2}}^{k},
$$

where $\omega_{k}$ is the nonlinear weight of stencil $S_{k}$. According to Jiang and Shu [49], the nonlinear weight is calculated by

$$
\omega_{k}=\frac{\alpha_{k}}{\sum_{k=0}^{r} \alpha_{k}}, \alpha_{k}=\frac{C_{k}}{\left(\varepsilon+\beta_{k}\right)^{p}} .
$$

$\beta_{k}$ is the smoothness measurement which becomes larger when discontinuities

are present within stencil $S_{k}$ and remains relatively small otherwise. The definition of $\beta_{k}$ presented by Jiang and Shu [49] is as follows

$$
\begin{aligned}
& \beta_{0}=\frac{13}{12}\left(\bar{q}_{j-2}-2 \bar{q}_{j-1}+\bar{q}_{j}\right)^{2}+\frac{1}{4}\left(\bar{q}_{j-2}-4 \bar{q}_{j-1}+3 \bar{q}_{j}\right)^{2}, \\
& \beta_{1}=\frac{13}{12}\left(\bar{q}_{j-1}-2 \bar{q}_{j}+\bar{q}_{j+1}\right)^{2}+\frac{1}{4}\left(\bar{q}_{j-1}-\bar{q}_{j+1}\right)^{2}, \\
& \beta_{2}=\frac{13}{12}\left(\bar{q}_{j}-2 \bar{q}_{j+1}+\bar{q}_{j+2}\right)^{2}+\frac{1}{4}\left(3 \bar{q}_{j}-4 \bar{q}_{j+1}+\bar{q}_{j+2}\right)^{2}, \\
& \beta_{3}=\frac{13}{12}\left(\bar{q}_{j+1}-2 \bar{q}_{j+2}+\bar{q}_{j+3}\right)^{2}+\frac{1}{4}\left(5 \bar{q}_{j+1}-8 \bar{q}_{j+2}+3 \bar{q}_{j+3}\right)^{2} .
\end{aligned}
$$


The smoothness measurement $\beta_{3}$ of fully downwind candidate is required to satisfy

$$
\beta_{3}=\max _{0 \leq k \leq 3} \beta_{k}
$$

The modified limiting procedure [50] is introduced to discourage unnecessary adaptation and to improve dissipation characteristics of the nonlinear reconstruction.

$$
\beta_{k}= \begin{cases}0, & R(\beta)<\alpha_{A L}, \\ \beta_{k}, & \text { otherwise }\end{cases}
$$

where

$$
R(\beta)=\frac{\max _{0 \leq k \leq 3} \beta_{k}}{\min _{0 \leq k \leq 3} \beta_{k}+\varepsilon} .
$$

The nonlinear reconstruction will be called MDCD-WENO scheme. For simulations in this paper, $\varepsilon=1.0 \times 10^{-10}$ and $p=1$ are adopted for MDCDWENO scheme.

\subsection{Riemann solver}

The Riemann problem comprised of the left and right state, $\mathbf{Q}^{L}$ and $\mathbf{Q}^{R}$, is solved by the HLLC Riemann solver [35, 36]. The numerical flux is obtained as

$$
\mathbf{F}^{H L L C}= \begin{cases}F^{L}, & \text { if } 0 \leq s^{L}, \\ F_{*}^{L}, & \text { if } s^{L}<0 \leq s_{*}, \\ F_{*}^{R}, & \text { if } s_{*}<0 \leq s^{R}, \\ F_{R}, & \text { if } s^{R}<0,\end{cases}
$$


where the flux with $\mathrm{K}=\mathrm{L}$ or $\mathrm{R}$ is given by

$$
\mathbf{F}^{K}=\left[\begin{array}{l}
\rho^{K} u_{n}^{K} \\
\left(\rho Y_{i}\right)^{K} u_{n}^{K} \\
\rho^{K} u_{1}^{K} u_{n}^{K}+p^{K} n_{1} \\
\rho^{K} u_{2}^{K} u_{n}^{K}+p^{K} n_{2} \\
\rho^{K} u_{3}^{K} u_{n}^{K}+p^{K} n_{3} \\
\left(p^{K}\left(\Gamma^{K}+1\right)+\frac{1}{2} \rho^{K} u_{j}^{K} u_{j}^{K}\right) u_{n}^{K} \\
\Gamma^{K} u_{n}^{K}
\end{array}\right]
$$

and

$$
\mathbf{F}_{*}^{K}=\frac{s^{K}-u_{n}^{K}}{s^{K}-s_{*}}\left[\begin{array}{l}
\rho^{K} s_{*} \\
\left(\rho Y_{i}\right)^{K} s_{*} \\
\rho^{K} u_{1}^{K} s_{*}+\frac{s^{K}-s_{*}}{s^{K}-u_{n}^{K}} p^{K} n_{1}+s^{K} n_{1} \rho^{K}\left(s_{*}-u_{n}^{K}\right) \\
\rho^{K} u_{2}^{K} s_{*}+\frac{s^{K}-s_{*}}{s^{K}-u_{n}^{K}} p^{K} n_{2}+s^{K} n_{2} \rho^{K}\left(s_{*}-u_{n}^{K}\right) \\
\rho^{K} u_{3}^{K} s_{*}+\frac{s^{K}-s_{*}}{s^{K}-u_{n}^{K}} p^{K} n_{3}+s^{K} n_{3} \rho^{K}\left(s_{*}-u_{n}^{K}\right) \\
\left(E^{K}+p^{K}\right) s_{*}+s^{K} \rho^{K}\left(s_{*}-u_{n}^{K}\right) s_{*} \\
\Gamma^{K} s_{*}
\end{array}\right] .
$$

The wave speeds are calculated as follows

$$
s^{L}=\min \left(u_{n}^{R O E}-c_{n}^{R O E}, u^{L}-c^{L}\right), s^{R}=\max \left(u_{n}^{R O E}+c_{n}^{R O E}, u^{R}+c^{R}\right)
$$

where $u_{n}^{R O E}$ and $c_{n}^{R O E}$ are Riemann averages:

$$
u_{n}^{R O E}=\frac{u_{n}^{L} \sqrt{\rho^{L}}+u_{n}^{R} \sqrt{\rho^{R}}}{\sqrt{\rho^{L}}+\sqrt{\rho^{R}}}, c^{R O E}=\frac{c^{L} \sqrt{\rho^{L}}+c^{R} \sqrt{\rho^{R}}}{\sqrt{\rho^{L}}+\sqrt{\rho^{R}}}
$$

and

$$
u_{n}^{K}=\mathbf{u}^{K} \cdot \mathbf{n}=u_{j}^{K} n_{j}
$$


with the velocity vector $\mathbf{u}^{K}=u_{1}^{K} \mathbf{i}+u_{2}^{K} \mathbf{j}+u_{3}^{K} \mathbf{k}$ and the unite outward normal vector $\mathbf{n}=n_{1} \mathbf{i}+n_{2} \mathbf{j}+n_{3} \mathbf{k}$. The wave speed in the star region is computed according to Batten et al. [51],

$$
s_{*}=\frac{p^{R}-p^{L}+\rho^{L} u_{n}^{L}\left(s^{L}-u_{n}^{L}\right)-\rho^{R} u_{n}^{R}\left(s^{R}-u_{n}^{R}\right)}{\rho^{L}\left(s^{L}-u_{n}^{L}\right)-\rho^{R}\left(s^{R}-u_{n}^{R}\right)} .
$$

The velocity $(\mathbf{u} \cdot \mathbf{n})^{H L L C}$ in the source term of the transport equation is computed by

$$
(\mathbf{u} \cdot \mathbf{n})^{H L L C}=u_{n}^{H L L C}= \begin{cases}u_{n}^{L}, & \text { if } 0 \leq s^{L} \\ u_{n}^{L}+s^{L}\left(\frac{s^{L}-u_{n}^{L}}{s^{L}-s_{*}}-1\right), & \text { if } s^{L}<0 \leq s_{*} \\ u_{n}^{R}+s^{R}\left(\frac{s^{R}-u_{n}^{R}}{s^{R}-s_{*}}-1\right), & \text { if } s_{*}<0 \leq s^{R} \\ u_{n}^{R}, & \text { if } s^{R}<0 .\end{cases}
$$

\section{Numerical results}

The following one- and two- dimensional test cases are used to validate and verify the proposed scheme. We compare the MDCD-WENO scheme with the MUSCL (Monotone Upstream-centered Schemes for Conservation Law) scheme [52], WENO-JS scheme [49] and WENO-SYMBO scheme [48]. Third order standard Runge-Kutta scheme is used for temporal discretization. All test cases are conducted by solving Euler equations.

\subsection{Convergence study}

The multi-species advection test case similar to Wong and Lele [37] is used to verify the formal order of accuracy and resolution of the proposed scheme. The ratios of specific heats are 1.4 and 1.66 for the two gases of 
Table 1: $L_{2}$ errors and orders of covergence of mass fraction for one-dimensional multimaterial advection problem from different schemes at $t=2.0$

\begin{tabular}{|c|c|c|c|c|c|c|c|c|}
\hline \multirow{2}{*}{ Number of cells } & \multicolumn{2}{|c|}{ MUSCL } & \multicolumn{2}{|c|}{ WENO-JS } & \multicolumn{2}{|c|}{ WENO-SYMBO } & \multicolumn{2}{|c|}{ MDCD-WENO $(0.01)$} \\
\hline & error & order & error & order & error & order & error & order \\
\hline 4 & $7.587 \mathrm{e}-02$ & & $7.012 \mathrm{e}-02$ & & $6.332 \mathrm{e}-02$ & & $3.688 \mathrm{e}-02$ & \\
\hline 8 & $6.135 \mathrm{e}-02$ & 0.307 & $1.174 \mathrm{e}-02$ & 2.579 & $3.489 \mathrm{e}-03$ & 4.182 & $1.743 \mathrm{e}-03$ & 4.403 \\
\hline 16 & $2.657 \mathrm{e}-02$ & 1.207 & $7.089 \mathrm{e}-04$ & 4.049 & $4.218 \mathrm{e}-04$ & 3.048 & $1.614 \mathrm{e}-04$ & 3.432 \\
\hline 32 & $1.074 \mathrm{e}-02$ & 1.307 & $2.644 \mathrm{e}-05$ & 4.745 & $1.905 \mathrm{e}-05$ & 4.468 & $1.105 \mathrm{e}-05$ & 3.869 \\
\hline 64 & $3.658 \mathrm{e}-03$ & 1.553 & $8.631 \mathrm{e}-07$ & 4.937 & $1.121 \mathrm{e}-06$ & 4.087 & $6.915 \mathrm{e}-07$ & 3.998 \\
\hline 128 & $1.153 \mathrm{e}-03$ & 1.666 & $3.223 \mathrm{e}-08$ & 4.743 & $5.541 \mathrm{e}-08$ & 4.339 & $2.814 \mathrm{e}-08$ & 4.619 \\
\hline
\end{tabular}

nitrogen and helium respectively. The ratio of specific heats is calculated according the mixing rule of Eqs.(6)-(7). The molecular mass of nitrogen is 28.0 while that of helium is 4.0 . The initial conditions are

$$
\left(\rho_{1}, \rho_{2}, u, p, Y_{1}\right)=\left(7.0,1.0,1.0,1 / \gamma_{N_{2}}, 0.5+0.25 \sin [\pi(x+0.5)]\right)
$$

The computational domain $x \in[-1.0,1.0]$ is discretized from $N=4$ to $N=128$ cells. Periodic boundary conditions are imposed at locations $x=$ 1.0. The solution is advanced in time up to $t=2.0$. CFL number of 0.1 is chosen to make the error due to spatial discretization dominant. The numerical errors in terms of $L_{2}$ norm are shown in Table 1 with different grid resolutions. The results indicate that all schemes can achieve the expected rates of convergence in smooth multi-species flows.

To assess the efficiency of the present scheme, the relations between $L_{2}$ errors, number of cells and the CPU time are shown in Fig. 2 for the present MDCD scheme and other schemes. In the comparison, the same computational conditions are used for all the calculations. The limiting procedure of MUSCL method for comparison is introduced following the one dimensional 

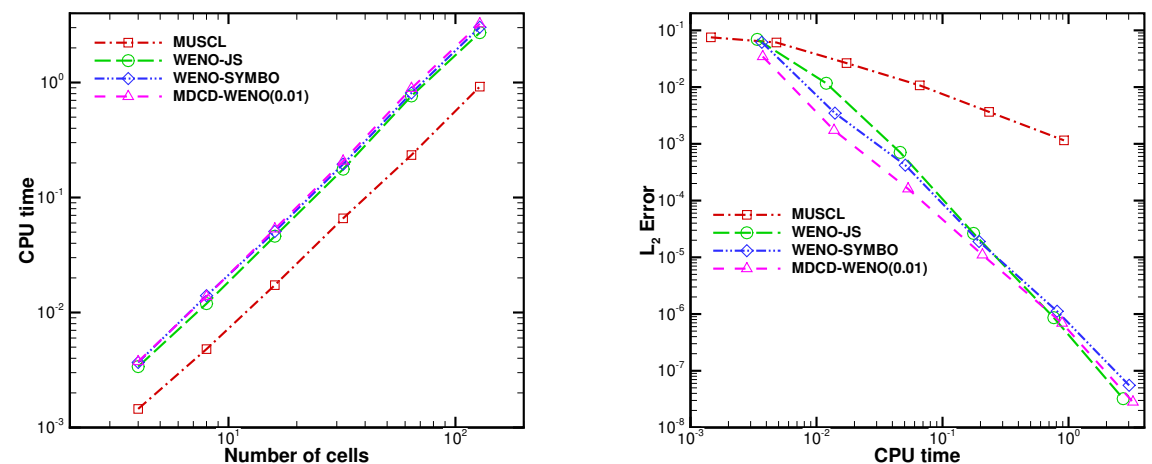

Figure 2: Comparisons of computational effort and accuracy with different numerical schemes.

case in Section 3.2. The left state $q_{j+\frac{1}{2}}^{L}$ and right state $q_{j-\frac{1}{2}}^{R}$ are computed by

$$
q_{j+\frac{1}{2}}^{L}=\bar{q}_{j}+\frac{\delta_{j+\frac{1}{2}}}{2} \varphi\left(\theta_{j}\right), q_{j-\frac{1}{2}}^{R}=\bar{q}_{j}-\frac{\delta_{j-\frac{1}{2}}}{2} \varphi\left(\frac{1}{\theta_{j}}\right),
$$

where $\delta_{j+\frac{1}{2}}=\bar{q}_{j+1}-\bar{q}_{j}, \theta_{j}=\delta_{j-\frac{1}{2}} / \delta_{j+\frac{1}{2}}$ and $\varphi\left(\theta_{j}\right)$ is the limiter:

$$
\varphi\left(\theta_{j}\right)= \begin{cases}\frac{\theta_{j}\left(\theta_{j}+2\right)}{2-\theta_{j}+2 \theta_{j}^{2}}, & \text { if } \theta_{j}>0 \\ 0, & \text { otherwise. }\end{cases}
$$

The reconstruction of MUSCL method can achieve second order of accuracy in smooth region and first order near discontinuities. The results demonstrate that for the same level of numerical error, the MDCD scheme spends less CPU time than MUSCL and WENO-SYMBO schemes; and using the same CPU time, the MDCD scheme predicts the solution with smaller error than MUSCL and WENO-SYMBO schemes. Because WENO-JS scheme has the highest order of accuracy (5th order), it will gradually become the most efficient scheme as increase of the grid resolution. As shown in Fig. 2, 
at least 64 cells are needed to make WENO-JS scheme more efficient than MDCD scheme. This is corresponding to 64 cells in one wavelength to resolve the highest wavenumber component of the mixture solution. In practical applications such as DNS of turbulent flow, one can rarely use such a large number of cells. If about 10-20 cells in one wavelength are used to compute the highest wavenumber component, the MDCD scheme will be several times more efficient than WENO-JS scheme.

As for the nonlinear problems with discontinuities, the high-order methods are subject to a loss of formal accuracy and may lose the advantage in the smooth region when the nonlinearity is introduced [53]. Compared to second-order method, the high-order scheme is more accurate but not accurate enough near the discontinuities to make up the difference in computational efforts due to the loss of formal accuracy [53]. Therefore, the efficiency of high-order methods in terms of error vs CPU effort may vary from linear to nonlinear problems. It is well-known that one of the bottlenecks for highorder numerical methods is the construction of shock capturing high-order limiters for nonlinear problems with discontinuities. However, one can expect that high-order methods will be more efficient for nonlinear problems with discontinuities when high-order limiters are developed to maintain high-order accuracy near the discontinuities.

\subsection{Advection of an isolated material interface}

This is an advection problem of a material interface and the material interface separates different gases of nitrogen and helium [21]. The values of velocity, pressure and temperature are constant across the material interface. This problem is used to test the capabilities of numerical schemes in 
maintaining the velocity, pressure and temperature equilibrium. The initial conditions are

$$
(\rho, u, p, \gamma, W)= \begin{cases}\left(7.0,1.0,1 / \gamma_{N_{2}}, 1.4,28.0\right)_{N_{2}}, & -0.5<x<0.5 \\ \left(1.0,1.0,1 / \gamma_{N_{2}}, 1.66,4.0\right)_{H e}, & \text { otherwise } .\end{cases}
$$

The computational domain $x \in[-1.0,1.0]$ is discretized to equally spacing
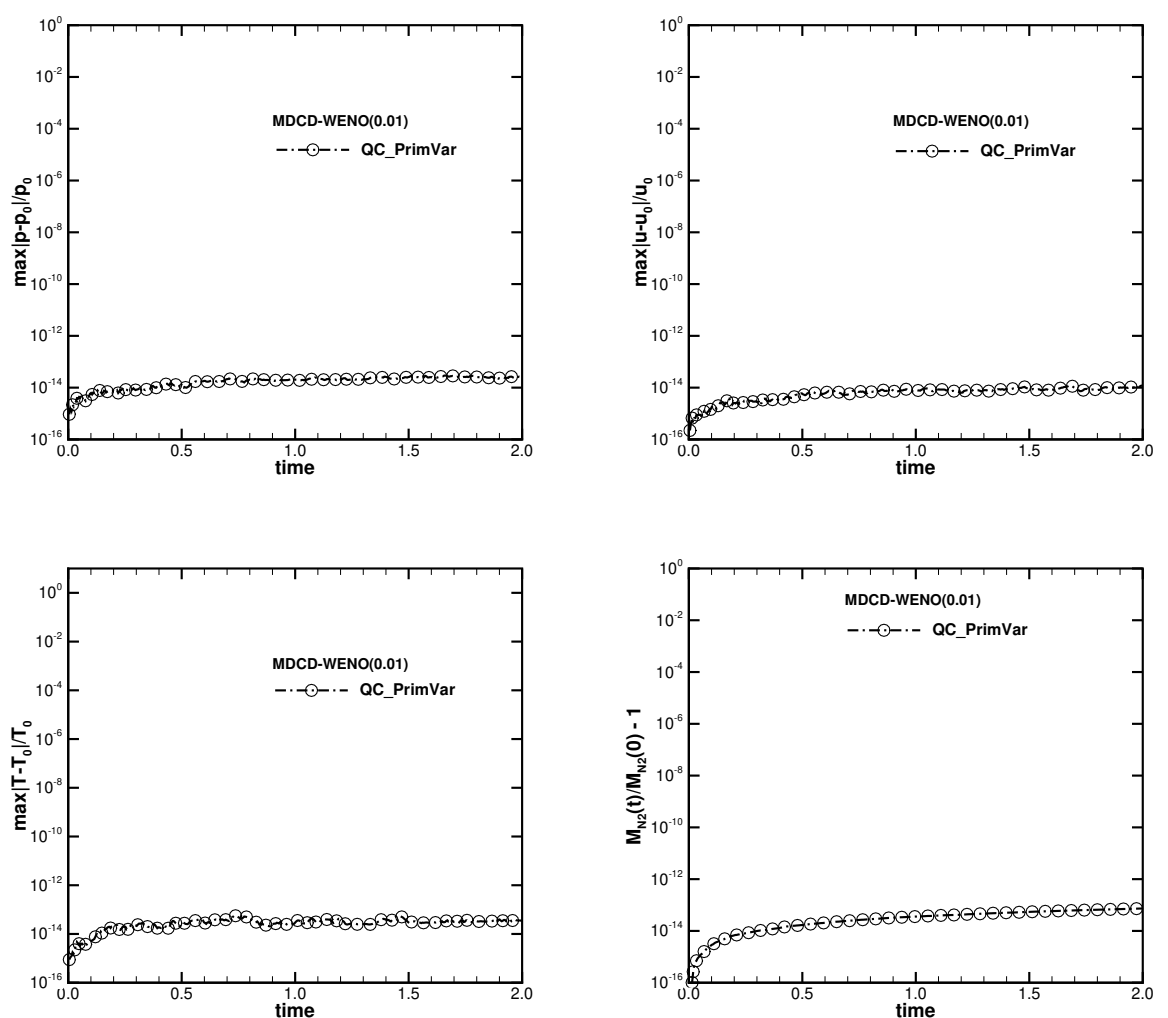

Figure 3: Time history of normalized errors for advection of a helium/nitrogen interface.

100 cells. Periodic boundary conditions are imposed at locations $x= \pm 1.0$. The solution is advanced in time up to $t=2.0$.

Fig. 3 shows the numerical errors for the quasi-conservative FV method based on MDCD-WENO reconstruction with primitive variables $\left(\rho, \rho Y_{i}, u_{j}, p, \Gamma\right)^{T}$. 

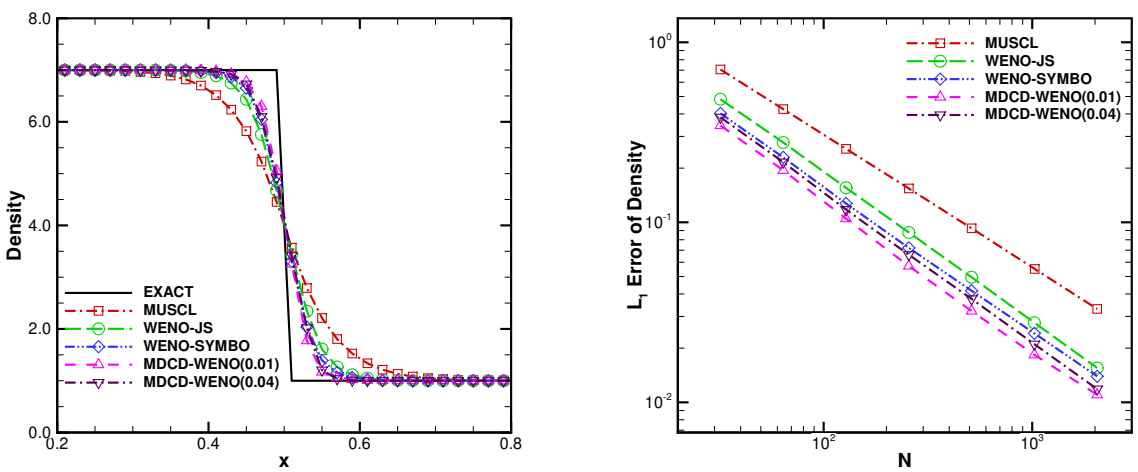

Figure 4: Density profiles and numerical errors of the advection of a material interface using different schemes.

As shown in Fig. 3, spurious oscillations for pressure, velocity and temperature are reduced to the order $O\left(10^{-13}\right)$ in round-off error. Furthermore, the present scheme can conserve the mass of nitrogen to round-off error.

In Fig. 4, the results obtained by MUSCL, WENO-JS, WENO-SYMBO and MDCD-WENO schemes are compared. The plots of density show that the present MDCD-WENO scheme improves resolution of the diffusive material interface and can capture the material interface more sharply than MUSCL, WENO-JS and WENO-SYMBO schemes.

The error norms as function of number of cell $N$ and comparison with other numerical schemes are shown in Fig. 4. The theoretical density profile is discontinuous across the material interface in this test case. Numerical errors of all numerical schemes decrease as grid resolution increased. With the same grid resolution, the MDCD-WENO scheme shows more accurate results compared to other schemes. 


\subsection{Advection of a contact discontinuity}

This is an advection problem of a contact discontinuity separating nitrogen and helium gases [21][39]. The velocity and pressure are constant while the temperature jumps across the material interface. The initial conditions are

$$
(\rho, u, p, \gamma, W)= \begin{cases}\left(14.0,1.0,1 / \gamma_{N_{2}}, 1.4,28.0\right)_{N_{2}}, & -0.5<x<0.5 \\ \left(1.0,1.0,1 / \gamma_{N_{2}}, 1.66,4.0\right)_{H e}, & \text { otherwise }\end{cases}
$$

The computational domain is $-1.0 \leq x \leq 1.0$ with equal grid spacing $h=0.02$. Periodic boundary conditions are imposed at locations $x= \pm 1.0$. The solution is advanced in time up to $t=2.0$.

Fig. 5 shows density and temperature profiles of the advection of a helium/nitrogen contact discontinuity after one period for MUSCL, WENOJS, WENO-SYMBO and MDCD-WENO schemes. The plots show that the present MDCD-WENO scheme with $\gamma_{\text {diss }}=0.01$ and $\gamma_{\text {diss }}=0.04$ improves resolution of the diffusive material interface and can capture the material interface more sharply than other schemes, as expected. Theoretical velocity and pressure profiles are constant across the material interface in this test case. The plots of velocity and pressure in Fig. 5 show that the applications of all schemes to the physical model are free of spurious oscillations.

Furthermore, the numerical errors of field variables of different schemes with mesh refinement from $N=32$ to $N=2048$ are shown in Fig. 6. Since the theoretical density and temperature profiles are discontinuous across the material interface in this test case, the numerical errors of density and temperature of different numerical schemes are to the order of the grid spacing $h$. On the other hand, the numerical errors of velocity and pressure for MDCD- 

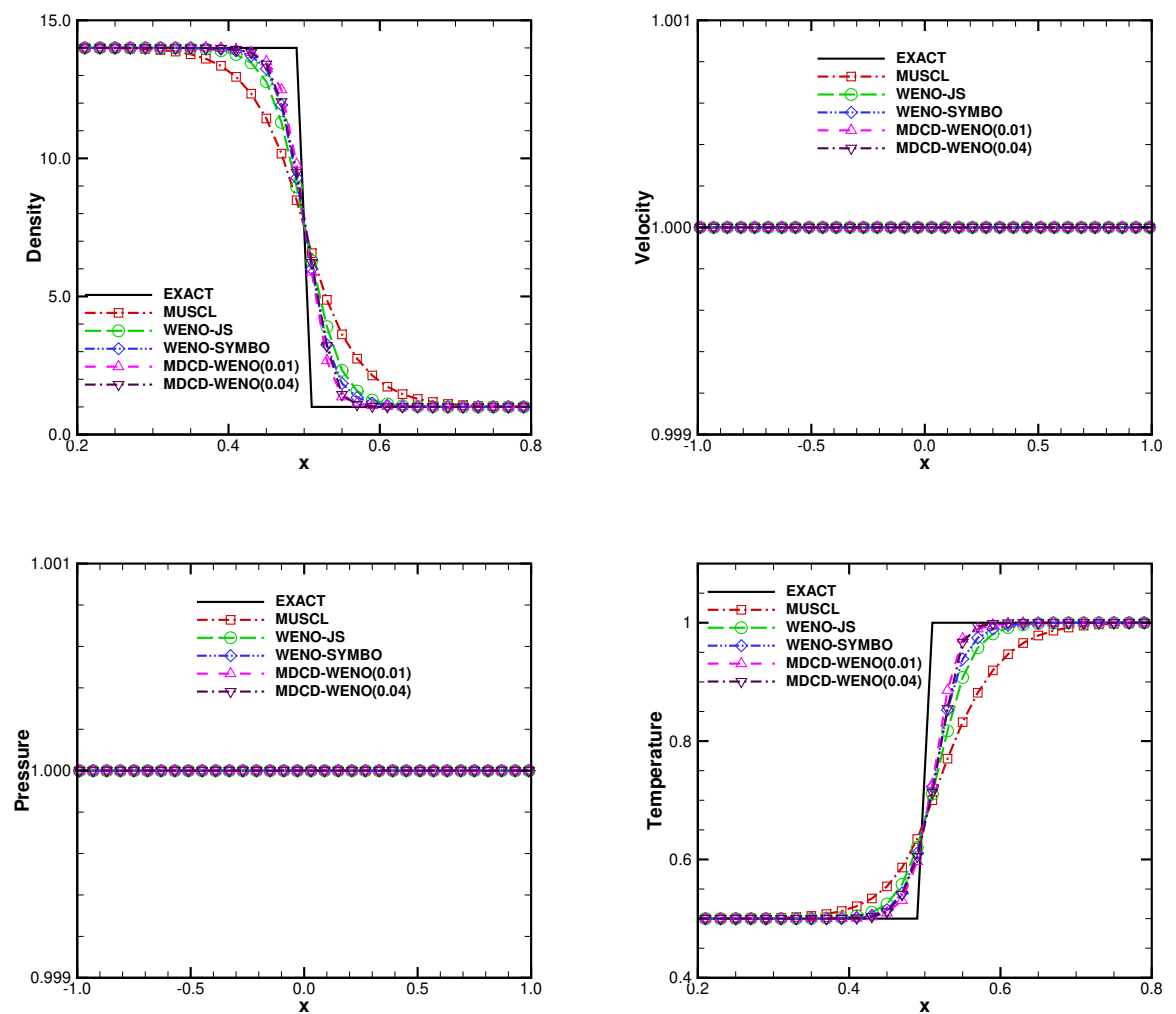

Figure 5: Profiles of the advection of a contact discontinuity with different numericalschemes.

WENO scheme are reduced to the order in round-off error with different grid resolutions due to free of spurious oscillations. In general, the results show that MDCD-WENO scheme can produce more accurate results compared to other numerical schemes.

\subsection{Shock tube problem}

This is a shock tube problem separating nitrogen and helium gases [21][39]. This problem is characterized by a strong shock wave, a rarefaction wave and 

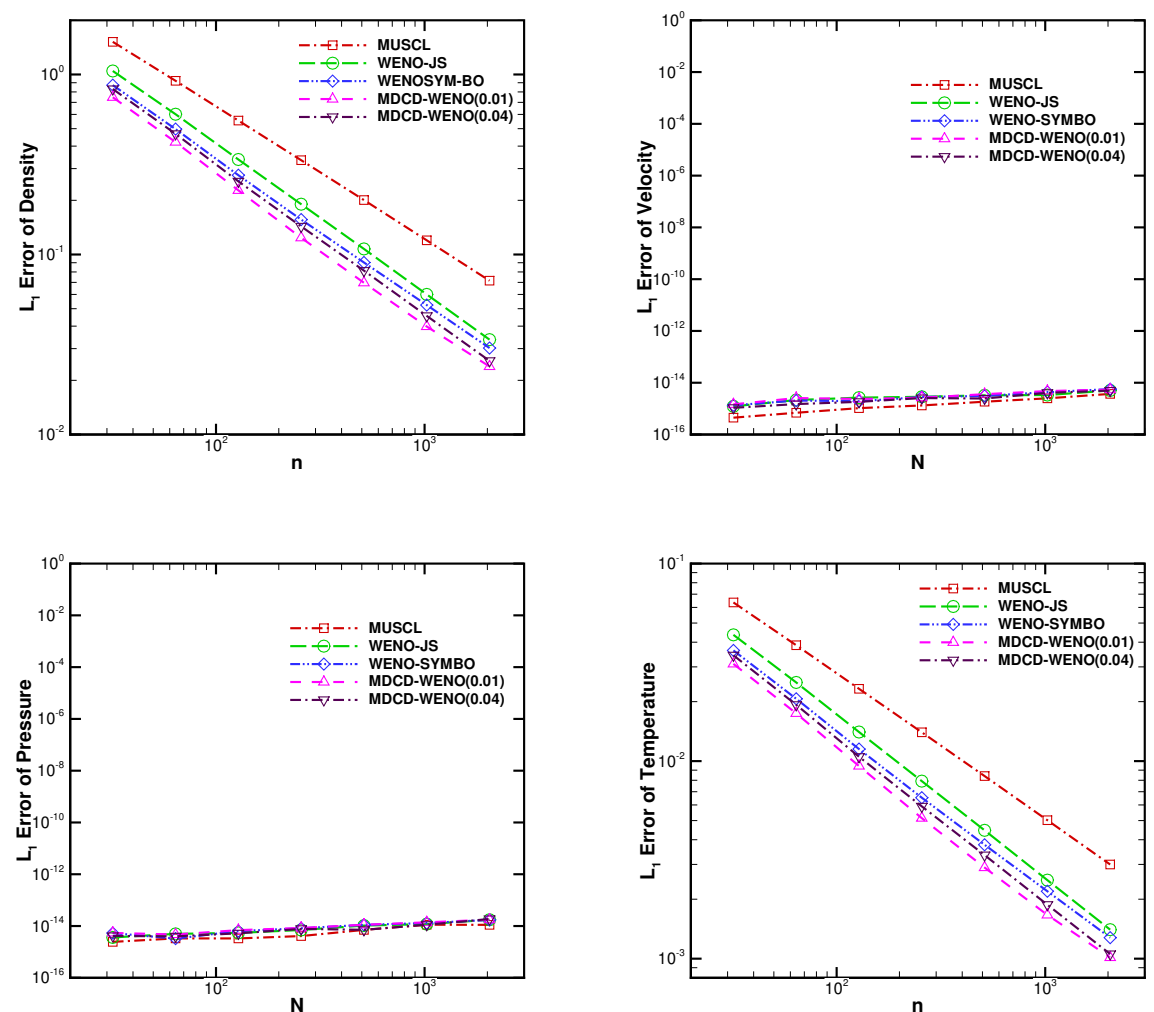

Figure 6: Numerical errors of field variables for different grid resolutions and with different numerical schemes.

a contact discontinuity. The initial conditions are

$$
(\rho, u, p, \gamma, W)= \begin{cases}\left(1.0,0.0,1 / \gamma_{N_{2}}, 1.4,28.0\right)_{N_{2}}, & 0.0<x<0.5 \\ \left(0.125,0.0,0.1 / \gamma_{N_{2}}, 1.66,4.0\right)_{H e}, & \text { otherwise. }\end{cases}
$$

The computational domain is $0.0 \leq x \leq 1.0$ with equal grid spacing $h=$ 0.0025. The material interface is at the location of $x=0.65$ when the simulation is at $t=0.2$. The maximum dissipation $\gamma_{\text {diss }}=0.046$ is adapted for MDCD-WENO scheme to provide sufficient numerical dissipation for the rarefaction waves. 

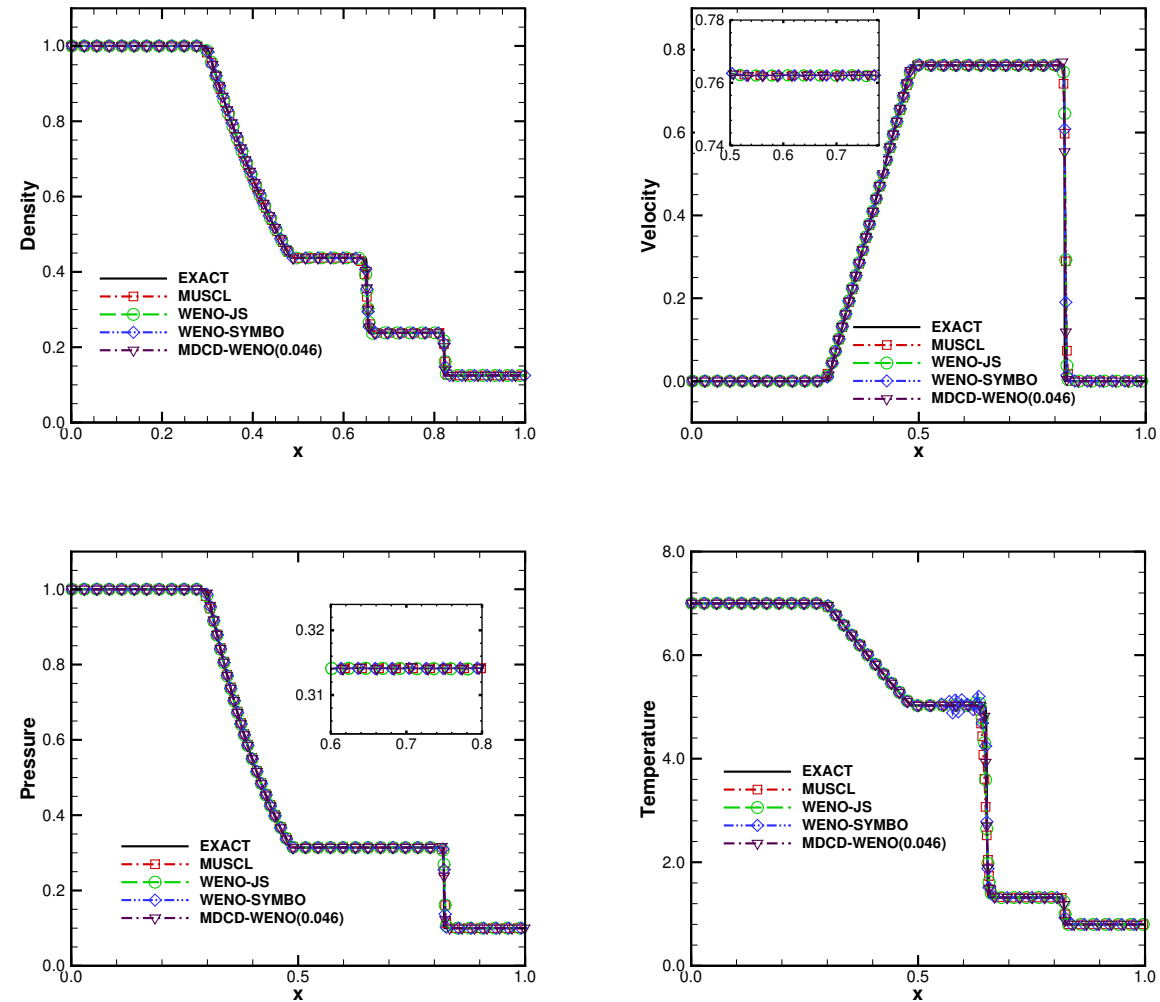

Figure 7: Profiles of a shock tube problem near the material interface with the present MDCD-WENO schemes and other numerical schemes.

The density, velocity, pressure and temperature profiles with details near the material interface obtained by MUSCL, WENO-JS, WENO-SYMBO and MDCD-WENO schemes are shown in Fig. 7 and Fig. 8. The exact results are calculated by MUSCL scheme with 10240 cells. The present MDCD-WENO scheme can capture the material interface and the temperature discontinuity more sharply and maintain the velocity and pressure equilibriums, compared to other schemes.

The numerical errors of different numerical schemes are shown in Fig. 9 

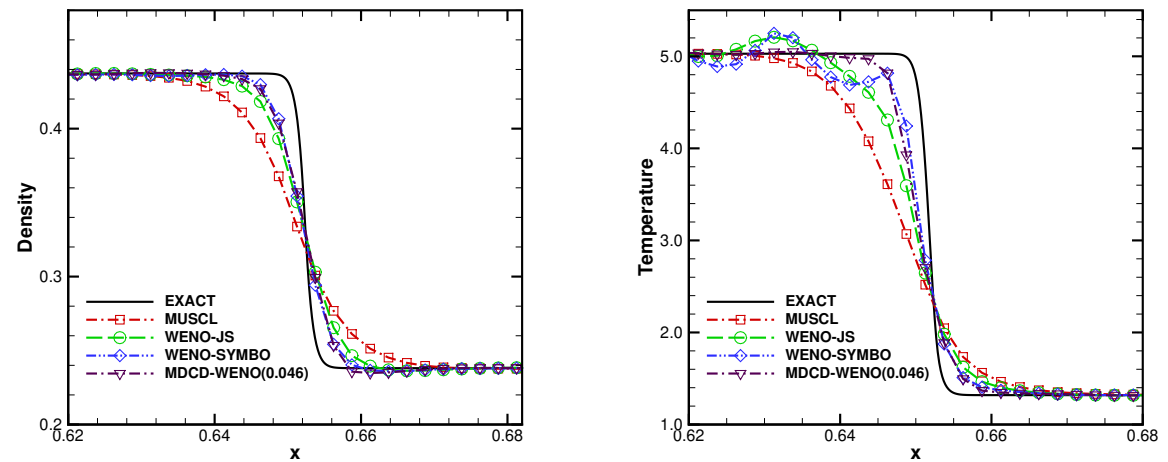

Figure 8: Local profiles of a shock tube problem near the material interface with different numerical schemes.

with mesh refinement from $N=32$ to $N=2048$. Generally, the MDCDWENO scheme can produce more accurate results than MUSCL, WENO-JS and WENO-SYMBO schemes with the same grid resolution.

\subsection{Two-dimensional Richtmyer-Meshkov instability}

The single-mode Richtmyer-Meshkov instability [39] is considered to illustrate the performance of WENO-JS, WENO-SYMBO and MDCD-WENO schemes. This problem is often utilized as a validation case for multi-material flow algorithms [21][39]. The material interface separating air from SF6 is impacted by a Mach 1.24 shock wave. The computational domain is $[0.0,4.0] \times[-0.5,0.5]$. The initial shape of the interface is given by

$$
x_{s}=2.9+0.1 \sin [2 \pi(y+0.25)],-0.5 \leq y \leq 0.5 .
$$



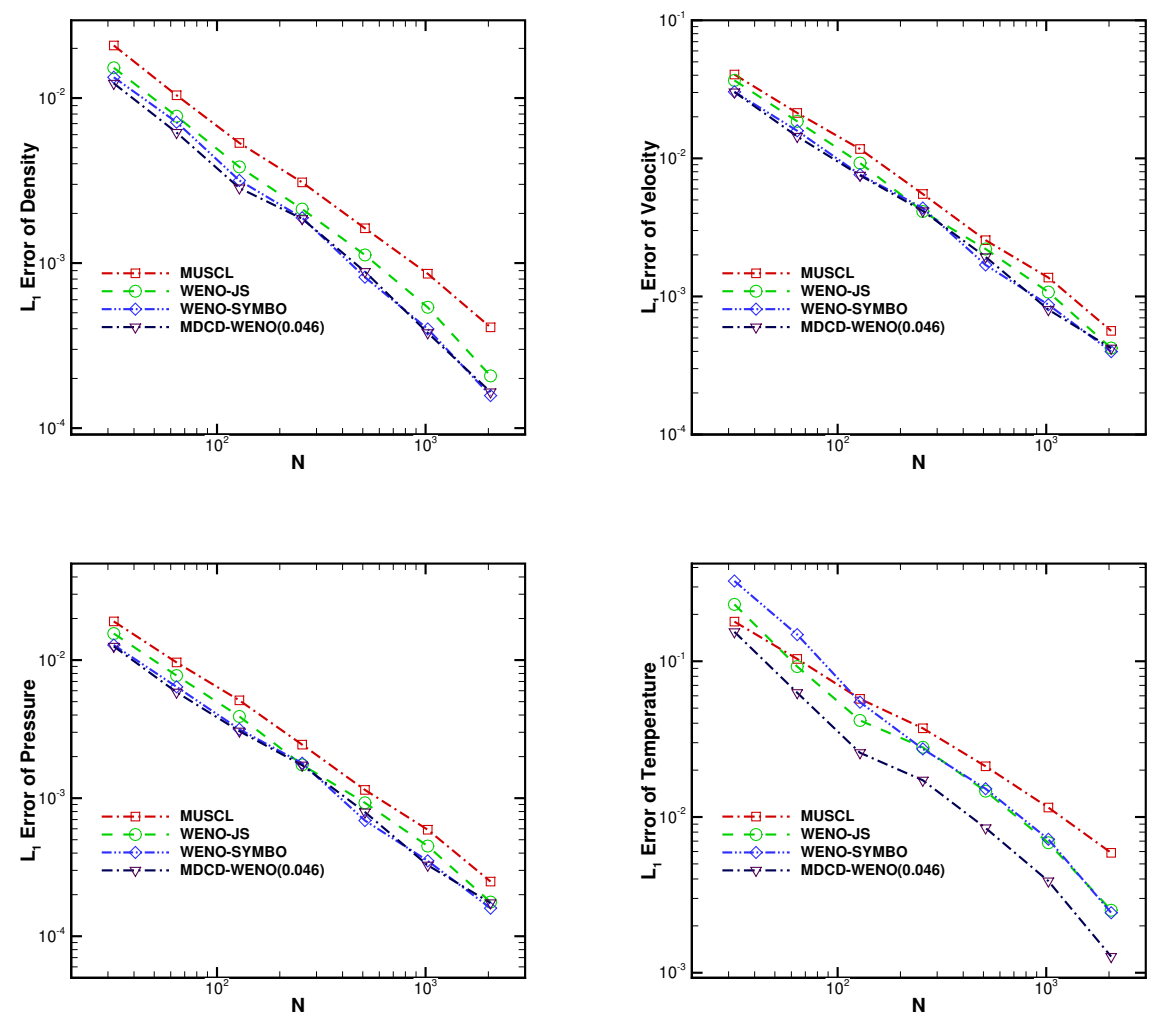

Figure 9: Numerical errors of field variables for different grid resolutions and with different numerical schemes.

The initial conditions are as follows:

$(\rho, u, v, p, \gamma, W)= \begin{cases}(5.0,0.0,0.0,1 / 1.4,1.093,144.0), & 0.0 \leq x \leq x_{s}, \\ (1.0,0.0,0.0,1 / 1.4,1.4,28.8), & x_{s}<x \leq 3.2, \\ (1.4112,-0.3613,0.0,1.6272 / 1.4,1.4,28.8), & 3.2<x \leq 4.0 .\end{cases}$

The variables are non-dimensionalized by the air density, acoustic velocity and temperature. The left moving Mach 1.24 shock wave is initially located at $x=3.2$. The periodic boundary conditions are imposed at the upper and lower boundaries. Simulations with different reconstruction schemes are 

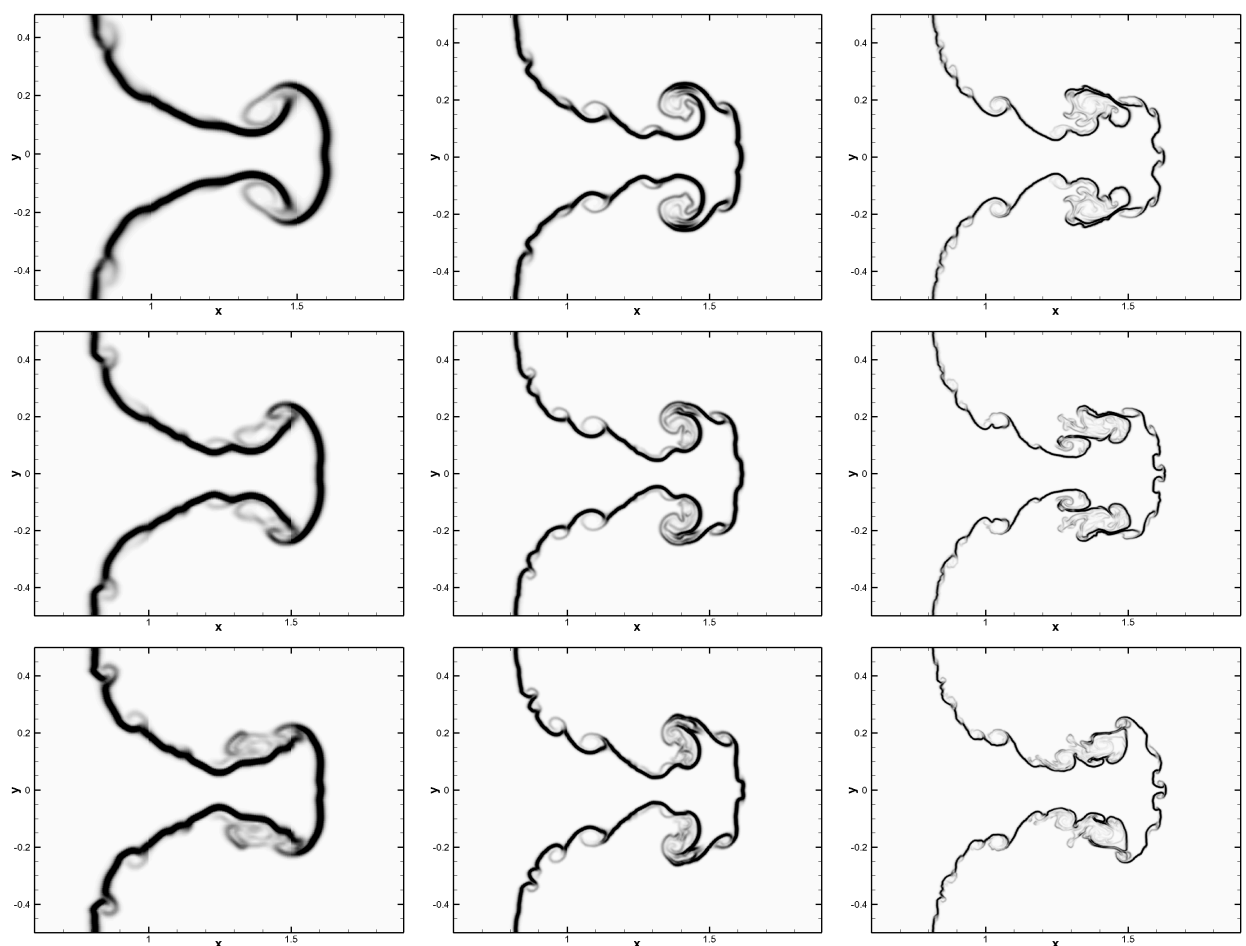

Figure 10: Comparison of interfaces using normalized density gradient magnitude $\phi=$ $\exp \left(|\nabla \rho| /|\nabla \rho|_{\max }\right)$ at $t=7.82 .50$ contours are from 1.0 to 1.5 using different schemes on coarse, intermediate and fine grids. Top row: WENO-JS; second row: WENO-SYMBO; Bottom row: MDCD-WENO.

carried up on three uniform grids, which are the coarse grid $(\Delta x=\Delta y=$ $1 / 128)$, medium grid $(\Delta x=\Delta y=1 / 256)$ and fine grid $(\Delta x=\Delta y=1 / 512)$. All runs are conducted with a constant CFL number of 0.32 .

Mushroom-shaped structures are developed from the vorticity generated by the interaction of the pressure gradient in the shock wave with the density gradient at the material interface. Since the inviscid simulations are conducted without physical dissipation, there is no convergent solutions with increasing the grid resolution. The instability of the bubble and spike is 

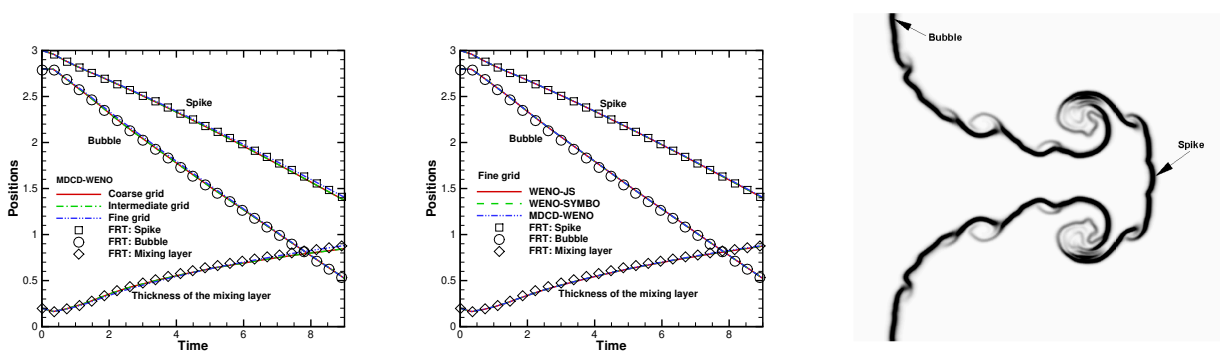

Figure 11: Time history of three characteristic positions and comparison with fronttracking method (FRT). Left column: Grid refinement test of MDCD-WENO scheme; Central column: Comparison of MDCD-WENO scheme with other numerical scheme on fine grid; Right column: Schematic of spike and bubble positions.

similar to the experimental images $[54,55]$ in the visual appearance. As the roll-up of vortical structures continues, the secondary instability along the interface produces local small-scale Kelvin-Helmholtz features. As Euler equations are solved for the test cases, the secondary instability features in fact are "numerical" Kelvin-Helmholtz instability. They are not found in the experiment $[54,55]$ due to the experimental diffusive nature of the initial perturbation [56] and that the experimental diffusion can smear small KelvinHelmholtz instabilities with sufficient physical dissipation. The generation of these numerical artifacts can be controlled by numerical dissipation[57, 58]. Therefore, finer structures are captured as the numerical order and resolution are increased [59]. As shown in Fig. 10, the MDCD-WENO scheme with less dissipation is capable to capture the smaller rolled up vortices near the interface, compared to other schemes.

At the early stage, the evolution for spike, bubble and the mixing layer (not for the small-scale interface structures) can be considered to be dominated by the primary instability due to large-scale baroclinic effects. Hence, 
they are weakly depending on the numerical methods and grid resolutions when the primary instability is resolved $[56,59]$. Fig. 11 shows the evolution of the location of the spike, the leading edge of the bubble, the thickness of the mixing layer and comparison with front-tracking/ghost-fluid method (FRT) [2]. The total mixing-layer thickness is defined as the distance between the spike and bubble. The grid refinement test of MDCD-WENO scheme and the comparison with other numerical schemes on the fine grid present mostly identical results for the locations of these three characteristic features. The results are in good agreement with the results of previous studies[2].

\subsection{Two-dimensional air-helium shock-cylinder interaction}

The two-dimensional shock-cylinder interaction problem [39] is simulated, which is associated with the interactions of a helium cylindrical bubble in air with a planar shock wave. This problem has been studied by many researcher

experimentally [60] and computationally [22]. Small-scale vortices near the interface developed due to baroclinic effects after the shock wave impact on the helium cylinder. This problem has been widely used to demonstrate the capabilities of capturing discontinuities and finer structures induced by instabilities along material interfaces.

The computational domain is $x \in[-3.5 D, 3.0 D]$ and $y \in[-0.9 D, 0.9 D]$, where $D$ denotes the diameter of the initial helium cylinder. Initially, the helium cylinder is located at $(0.0,0.0)$ and the left moving Mach 1.22 shock wave is placed at the location $x=1.0 D$. The initial conditions are given as $(\rho, u, v, p, \gamma, W)= \begin{cases}(1.3764,-0.3336,0.0,1.5698 / 1.4,1.4,28.8), & \text { post-shock } \\ (0.1819,0.0,0.0,1 / 1.4,1.648,4.0), & \text { bubble }, \\ (1.0,0.0,0.0,1 / 1.4,1.4,28.8), & \text { pre-shock }\end{cases}$ 

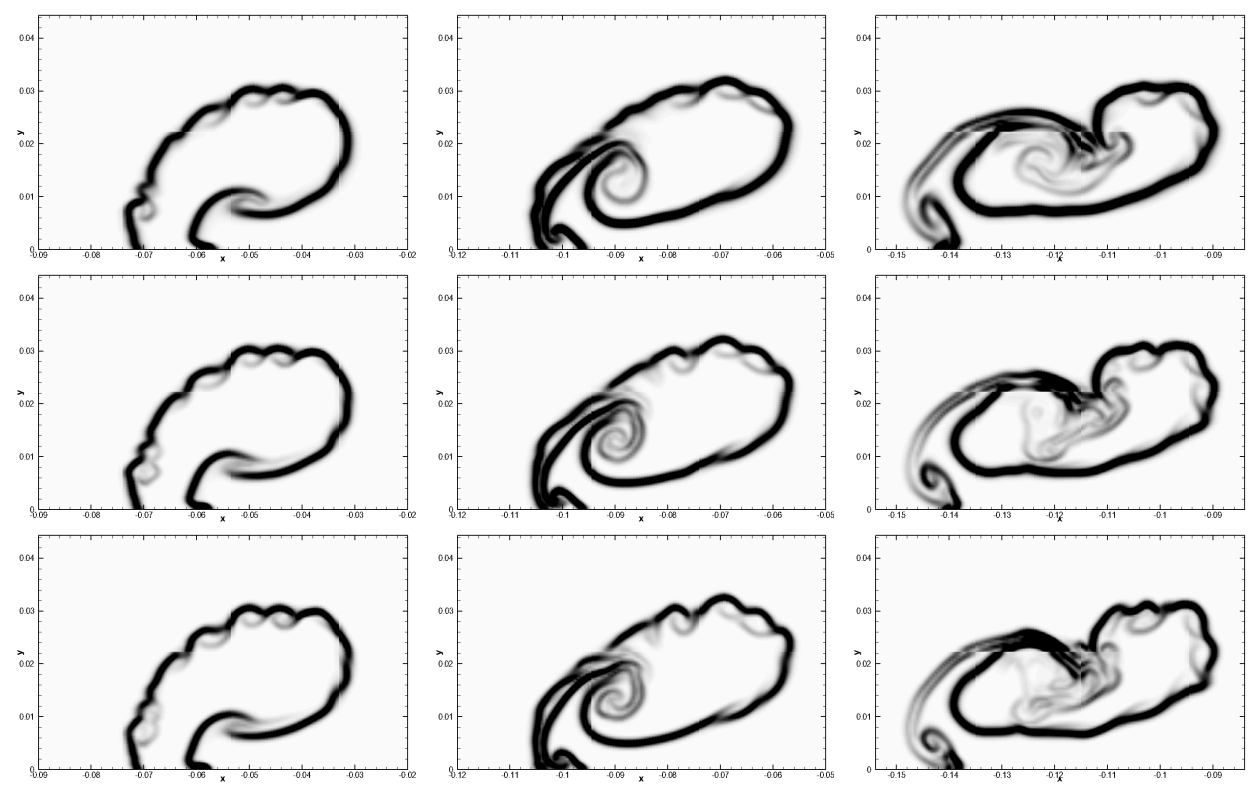

Figure 12: Comparison of the time evolution of the interface using normalized density gradient magnitude $\phi=\exp \left(|\nabla \rho| /|\nabla \rho|_{\max }\right)$ with 50 contours from 1.0 to 1.5 for the two-dimensional shock-cylinder interaction problem of various schemes. Grid resolution is $\Delta x=\Delta y=0.01 D$. Top row: WENO-JS; the second row: WENO-SYMBO; bottom row: MDCD-WENO. Left column: $t=480 \mu s$; central column: $t=720 \mu s$; right column: $t=1020 \mu s$.

with the initial diameter $D$ of $50 \mathrm{~mm}$. The pre-shock air quantities are used as reference. The slip wall boundary conditions are imposed at the upper and lower boundaries. All simulations are conducted with a constant CFL number of 0.32. Three levels of grid resolution $\Delta x=\Delta y=D / 100$, $\Delta x=\Delta y=D / 200$ and $\Delta x=\Delta y=D / 400$ with grid cells $650 \times 180$, $1300 \times 360$ and $2600 \times 720$ are employed. The normalized density gradient magnitude $\phi=\exp \left(|\nabla \rho| /|\nabla \rho|_{\max }\right)$ of different schemes with the effects of grid resolution is shown in Fig. 12 to Fig. 14.

There is no convergent solution when increasing grid resolution as this 

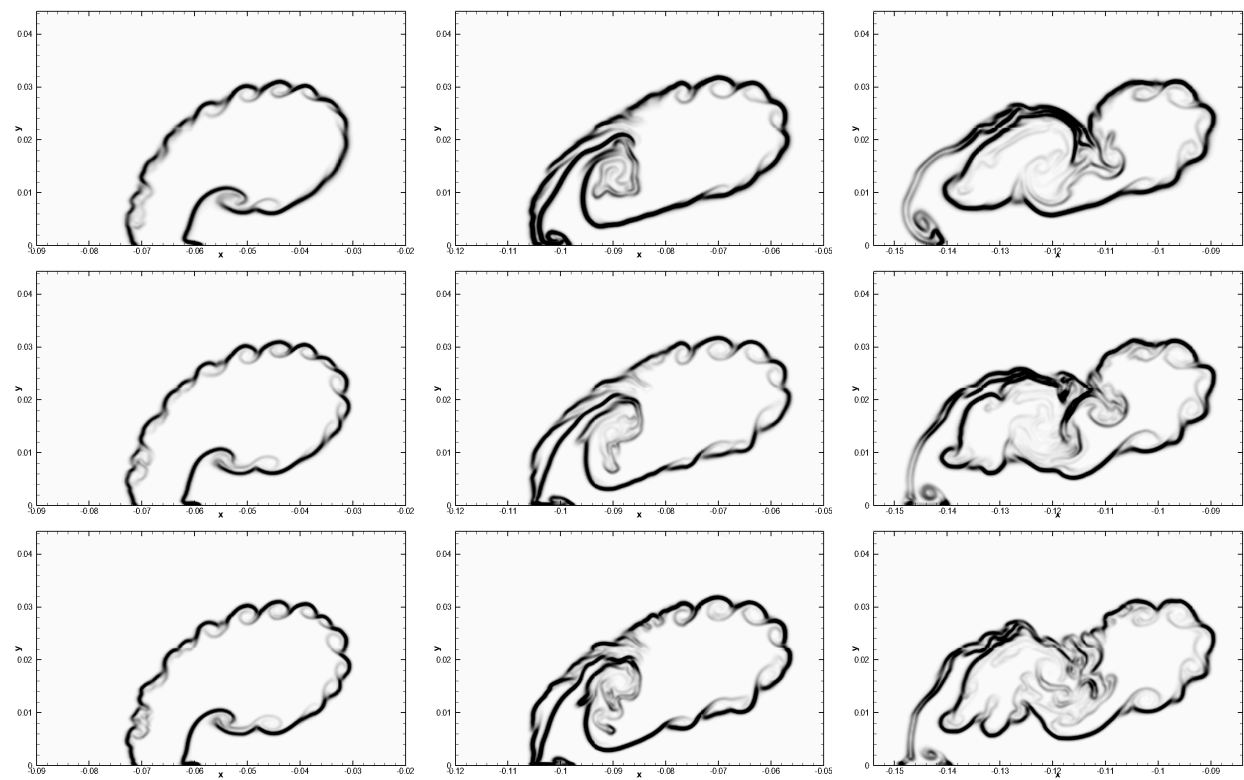

Figure 13: Comparison of the time evolution of the interface using normalized density gradient magnitude $\phi=\exp \left(|\nabla \rho| /|\nabla \rho|_{\max }\right)$ with 50 contours from 1.0 to 1.5 for the two-dimensional shock-cylinder interaction problem of various schemes. Grid resolution is $\Delta x=\Delta y=0.005 D$. Top row: WENO-JS; the second row: WENO-SYMBO; bottom row: MDCD-WENO. Left column: $t=480 \mu s$; central column: $t=720 \mu s$; right column: $t=1020 \mu s$.

inviscid test case is solved by Euler equations. According to the vorticity equation, the baroclinically generated vorticity is initially deposited in an approximately material interface. The spurious numerical oscillations regarded as the "baroclinic" source along the interface can induce spurious eddies[57, 58]. With physically consistent treatment of the reconstruction process and calculation of numerical flux by Riemann solver, there is no spurious oscillations of pressure arising from the interfaces as shown in Fig. 15 . Therefore, the "baroclinic" source of spurious eddies along the interface is eliminated. 

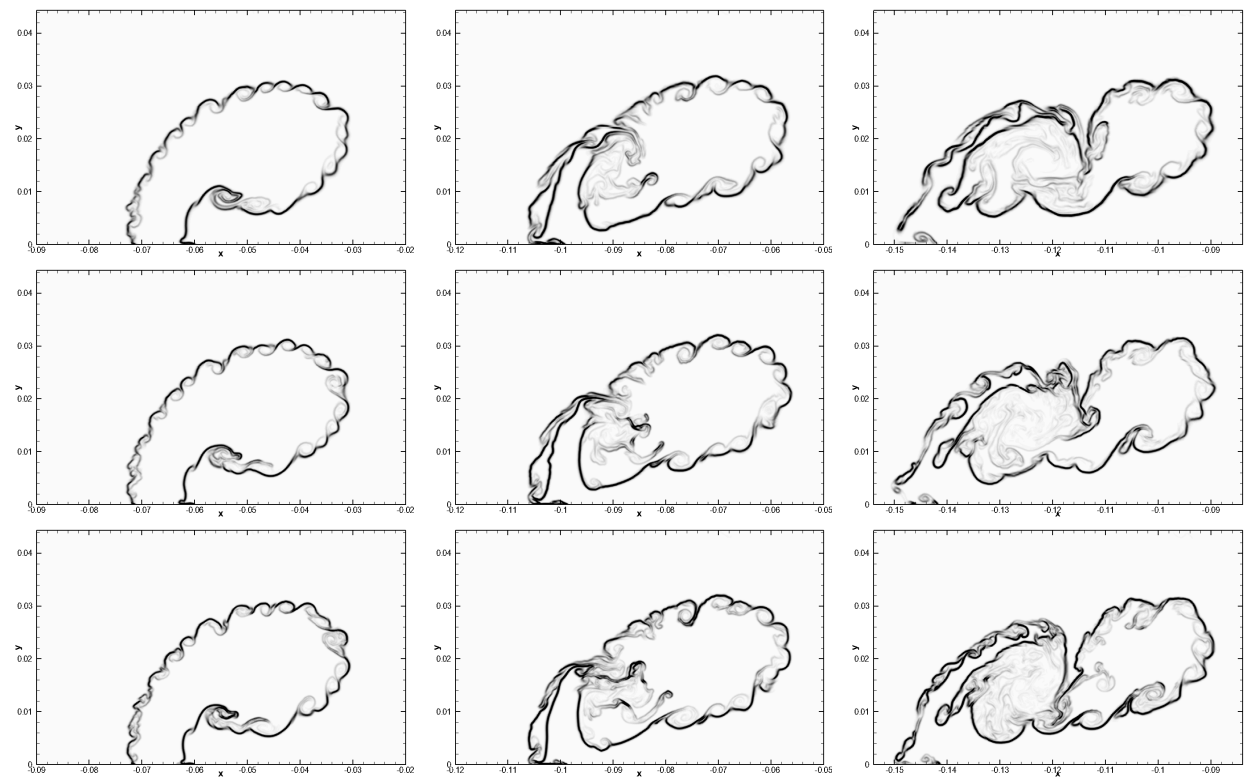

Figure 14: Comparison of the time evolution of the interface using normalized density gradient magnitude $\phi=\exp \left(|\nabla \rho| /|\nabla \rho|_{\max }\right)$ with 50 contours from 1.0 to 1.5 for the two-dimensional shock-cylinder interaction problem of various schemes. Grid resolution is $\Delta x=\Delta y=0.0025 D$. Top row: WENO-JS; the second row: WENO-SYMBO; bottom row: MDCD-WENO. Left column: $t=480 \mu s$; central column: $t=720 \mu s$; right column: $t=1020 \mu s$.

When the shock wave slides along the interfaces, vortical structures are established. As the roll-up continues, secondary instability of Kelvin-Helmonholtz features starts to manifest which disturb the smooth interfaces and form several small-scale vortex coils along the interfaces at the early stage as shown in Fig. 12 to Fig. 14 at $t=0.000480 s$. As these small vortex coils grow, the Kelvin-Helmonholtz features eventually breakdown into turbulently mixing area. In this inviscid shock-bubble interaction test case, no physical viscosity or diffusion is carried out to diffuse the small-scale Kelvin-Helmonholtz instabilities. Therefore, the resolution of the small-scale Kelvin-Helmonholtz 

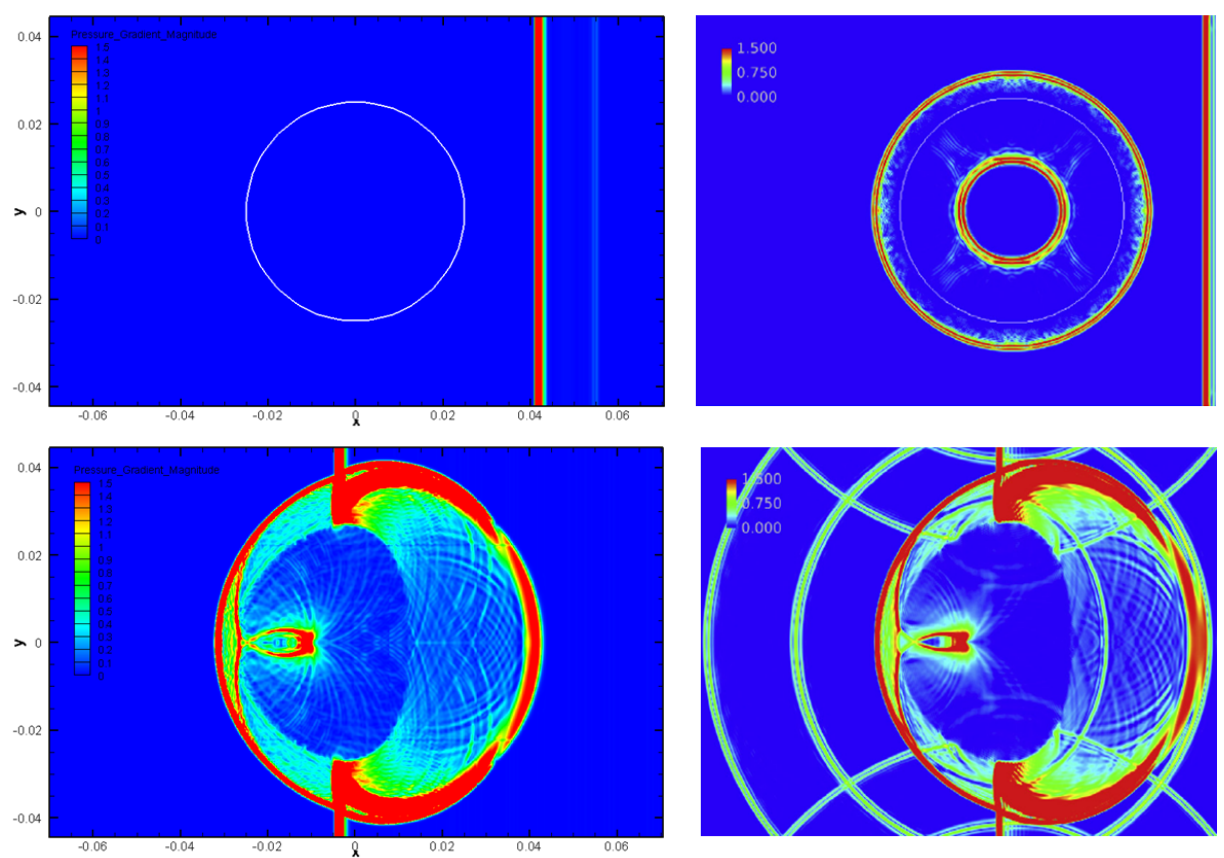

Figure 15: Comparison of the pressure gradient magnitude from 0.0 to 1.5 between the present method and FC method[39] during the early stage. The whit line shows the initial bubble surface. Left column: the present method with MDCD-WENO scheme; Right column: FC method.

features depends on the numerical viscosity introduced by dissipation property of the numerical scheme. When the grid resolution is increased, the small-scale features are produced. The high resolution scheme with low dissipation is capable to capture the smaller scales structures, as expected. As shown in Fig. 12 to Fig. 14, the MDCD-WENO scheme is less dissipative and then produces more small scale structures along the material interface than WENO-JS and WENO-SYMBO schemes.

The small bubbles at the top of the spike are produced. This is due to how to set the initial perturbation on the Cartesian grid. Hence, compared to 

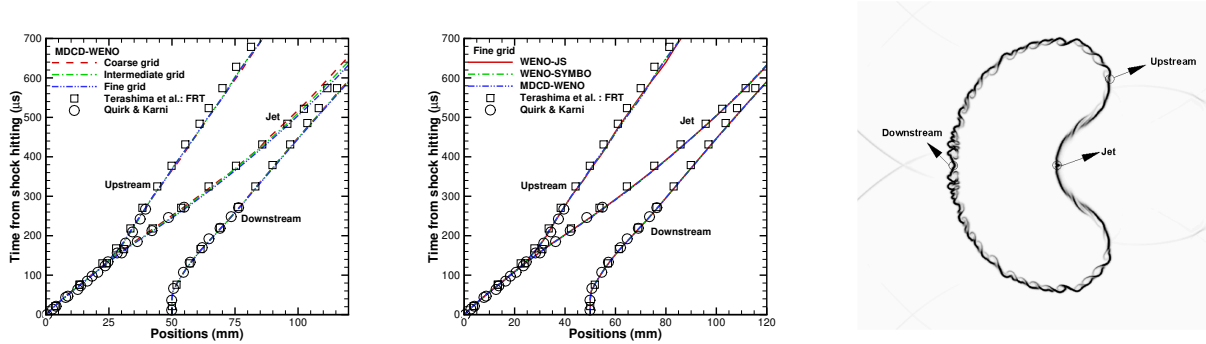

Figure 16: Space-time diagrams for three characteristic positions and comparison with front-tracking method (FRT)[2] and earlier studies [22]. Left column: Grid refinement tests of MDCD-WENO scheme; Central column: Comparison of MDCD-WENO scheme with other numerical scheme on fine grid; Right column: Schematic of three charateristic positions on interface.

the smooth initial condition due to experimental diffusion, the initial sharp discontinuity to the level of the resolution of the grid in simulations will introduce artificial disturbances and then induce secondary instabilities along the interface. These small bubbles at the top of the spike are sensitive to the grid resolution and numerical schemes. However, these instability features have no profound effects on the formulation of the vortices as shown in Fig. 12 to Fig. 14.

Fig. 16 shows space-time diagrams for three characteristic positions and comparison with front-tracking/ghost-fluid method (FRT) [2]. The grid refinement test of MDCD-WENO scheme and the comparison of the other schemes on the fine grid show mostly identical results for the locations of these three characteristic features. The present results are in good agreement with the results reported in earlier studies [2, 22, 39]. This is due to that the grid resolution and numerical schemes have little impact on the large scale features which are dominated by inviscid baroclinic effects. 


\section{Conclusion}

In this paper, the consistent high resolution FV method based on reconstruction with MDCD-WENO scheme is implemented for simulations of compressible multi-material flows. The proposed FV method discretely conserve mass of species, along with the total mass, momentum and energy, and maintains the velocity, pressure and temperature equilibriums to suppress spurious oscillations near material interfaces which may contaminate small-scale physical phenomena. Compared with MUSCL, WENO-JS and WENO-SYMBO schemes, the MDCD-WENO scheme can more sharply capture material interfaces and improve the resolution of finer structures with a broad range of length scales due to its optimized spectral properties. Moreover, the MDCD-WENO scheme with one more fully downwind stencil in addition to WENO-JS stencils is no more difficult than the implementations of WENO scheme for compressible multi-material flows. Several one- and two-dimensional problems with various discontinuities are used to verify the consistency and high resolution properties of the present scheme for simulations of compressible multi-material flows.

\section{Acknowledgements}

Most of this work was performed when the first author was at the University of Southampton as a postdoctoral researcher sponsored by China Scholarship Council (File No.201704890001). This research was supported by the National Natural Science Foundation of China (Grant No.11602029). This work used high performance clusters Iridis of the University of Southampton. 


\section{References}

[1] R. Saurel, F. Petitpas, P. Berry, Simple and efficient relaxation methods for interfaces separating compressible fluids, cavitating flows and shocks in multiphase mixtures, Journal of Computational Physics 228 (2009) $1678-1712$.

[2] H. Terashima, G. Tryggvason, A front-tracking/ghost-fluid method for fluid interfaces in compressible flows, Journal of Computational Physics 228 (2009) 4012-4037.

[3] C. W. Hirt, B. D. Nichols, Volume of fluid (VOF) method for the dynamics of free boundaries, Journal of Computational Physics 39 (1981) $201-225$.

[4] S. Osher, R. Fedkiw, Level set methods: an overview and some recent results, Journal of Computational Physics 169 (2001) 463-502.

[5] I.-L. Chern, J. Glimm, O. McBryan, B. Plohr, S. Yaniv, Front tracking for gas dynamics, Journal of Computational Physics 62 (1986) 83-110.

[6] T. Fedkiw, T. Aslam, B. Merriman, S. Osher, A non-oscillatory Eulerian approach to interfaces in multimaterial flows (the ghost fluid method), Journal of Computational Physics 152 (1999) 457-492.

[7] D.-K. Mao, Towards front tracking based on conservation in two space dimensions, SIAM Journal on Scientific Computing 22 (2000) 113-151.

[8] D.-K. Mao, Towards front tracking based on conservation in two space 
dimensions II, tracking discontinuities in capturing fashion, Journal of Computational Physics 226 (2007) 1550-1588.

[9] M. A. Ullah, W. B. Gao, D.-K. Mao, Towards front tracking based on conservation in two space dimensions III, tracking interfaces, Journal of Computational Physics 242 (2013) 268-303.

[10] P. T. Barton, B. Obadia, D. Drikakis, A conservative level-set based method for compressible solid/fluid problem on fixed grids, Journal of Computational Physics 230 (2011) 7867-7890.

[11] R. Saurel, C. Pantano, Diffuse-interface capturing methods for compressible two-phase flows, Annual Review of Fluid Mechanics 50 (2018) $105-130$.

[12] M. R. Baer, J. W. Nunziato, A two-phase mixture theory for the deflagration-to-detonation transition (DDT) interactive granular materials, International Journal of Multiphase Flow 12 (1986) 861-889.

[13] A. K. Kapila, R. Menikoff, J. B. Bdzil, S. F. Son, D. S. Stewart, Twophase modeling of deflagration-to-detonation transition in granular materials: Reduced equations, Physics of Fluids 13 (2001) 3002-3024.

[14] G. Allaire, S. Clerc, S. Kokh, A five-equation model for the simulation of interfaces between compressible fluids, Journal of Computational Physics 181 (2002) 577-616.

[15] A. Murrone, H. Guillard, A five equation reduced model for compressible two phase flow problems, Journal of Computational Physics 202 (2005) 664-698. 
[16] R. Abgrall, V. Perrier, Asymptotic expansion of a multiscale numerical scheme for compressible multiphase flow, multiscale model, Multiscale Modeling and Simulation 5 (2006) 84-115.

[17] Z.-S. Sun, Y.-X. Ren, L. Cédric, S.-Y. Zhang, Y.-C. Yang, A class of finite difference schemes with low dispersion and controllable dissipation for DNS of compressible turbulence, Journal of Computational Physics 230 (2011) 4616-4635.

[18] Q.-J. Wang, Y.-X. Ren, Z.-S. Sun, Y.-T. Sun, Low dispersion finite volume scheme based on reconstruction with minimized dispersion and controllable dissipation, Science China Physics, Mechanics and Astronomy 56 (2013) 423-431.

[19] R. Saurel, E. Franquet, E. Daniel, O. Le Metayer, A relaxation- projection method for compressible flows. Part I: The numerical equation of state for the Euler equations, Journal of Computational Physics 223 (2007) 822-845.

[20] S. Karni, Multi-component flow calculations by a consistent primitive algorithm, Journal of Computational Physics 112 (1994) 31-43.

[21] E. Johnsen, F. Ham, Preventing numerical errors generated by interfacecapturing schemes in compressible multi-material flows, Journal of Computational Physics 231 (2012) 5705-5717.

[22] J. J. Quirk, S. Karni, On the dynamics of a shock-bubble interaction, Journal of Fluid Mechanics 318 (1996) 129-163. 
[23] R. Abgrall, How to prevent pressure oscillations in multicomponent flow calculations: a quasi-conservative approach, Journal of Computational Physics 125 (1996) 150-160.

[24] R. Abgrall, S. Karni, Computations of compressible multifluids, Journal of Computational Physics 169 (2001) 594-623.

[25] K.-M. Shyue, An efficient shock-capturing algorithm for compressible multicomponent problems, Journal of Computational Physics 142 (1998) 208-242.

[26] S. Alahyari Beig, E. Johnsen, Maintaining interface equilibrium conditions in compressible multiphase flows using interface capturing, Journal of Computational Physics 302 (2015) 548-566.

[27] B. Thornber, M. Groom, D. L. Youngs, A five-equation model for the simulation of miscible and viscous compressible fluids, Journal of Computational Physics 372 (2018) 256-280.

[28] E. Olsson, G. Kreiss, S. Zahedi, A conservative level set method for two phase flow II, Journal of Computational Physics 225 (2007) 785-807.

[29] R. K. Shukla, C. Pantano, J. B. Freund, An interface capturing method for the simulation of multi-phase compressible flows, Journal of Computational Physics 229 (2010) 7411-7439.

[30] A. Tiwari, J. B. Freund, C. Pantano, A diffuse interface model with immiscibility preservation, Journal of Computational Physics 252 (2013) 290-309. 
[31] R. K. Shukla, Nonlinear preconditioning for efficient and accurate interface capturing in simulation of multicomponent compressible flows, Journal of Computational Physics 276 (2014) 508-540.

[32] K. K. So, X. Y. Hu, N. A. Adams, Anti-diffusion interface sharpening technique for two phase compressible flow simulations, Journal of Computational Physics 231 (2012) 4304-4323.

[33] K.-M. Shyue, F. Xiao, An Eulerian interface sharpening algorithm for compressible two-phase flow: the algebraic THINC approach, Journal of Computational Physics 268 (2014) 326-354.

[34] A. Chiapolino, R. Saurel, B. Nkonga, Sharpening diffuse interfaces with compressible fluids on unstructured meshes, Journal of Computational Physics 340 (2017) 389-417.

[35] E. Johnsen, T. Colonius, Implementation of WENO schemes in compressible multicomponent flows, Journal of Computational Physics 219 (2006) 715-732.

[36] V. Coralic, T. Colonius, Finite-volume WENO scheme for viscous compressible multicomponent flows, Journal of Computational Physics 274 (2014) 95-121.

[37] M. L. Wong, S. K. Lele, High-order localized dissipation weighted compact nonlinear scheme for shock-and interface-capturing in compressible flows, Journal of Computational Physics 339 (2017) 179-209.

[38] T. Nonomura, S. Morizawa, H. Terashima, S. Obayashi, K. Fujii, Numerical (error) issues on compressible multicomponent flows using a high- 
order differencing scheme: Weighted compact nonlinear scheme, Journal of Computational Physics 231 (2012) 3181-3210.

[39] H. Terashima, S. Kawai, M. Koshi, Consistent numerical diffusion terms for simulating compressible multicomponent flows, Computers and Fluids 88 (2013) 484-495.

[40] Z.-W. He, Y.-S. Zhang, X.-L. Li, L. Li, B.-L. Tian, Preventing numerical oscillations in the flux-split based finite difference method for compressible flows with discontinuities, Journal of Computational Physics 300 (2015) 269-287.

[41] Z.-W. He, Y.-S. Zhang, X.-L. Li, B.-L. Tian, Preventing numerical oscillations in the flux-split based finite difference method for compressible flows with discontinuities, II, International Journal for Numerical Methods in Fluids 80 (2016) 306-316.

[42] T. Nonomura, K. Fujii, Characteristic finite-difference WENO scheme for multicomponent compressible fluid analysis: Overestimated quasiconservative formulation maintaining equilibriums of velocity, pressure, and temperature, Journal of Computational Physics 340 (2017) 358-388.

[43] Z.-W. He, L. Li, Y.-S. Zhang, B.-L. Tian, Consistent implementation of characteristic flux-split based finite difference method for compressible multi-material gas flows, Computers and Fluids 168 (2018) 190-200.

[44] R. Vichnevetsky, J. B. Bowles, Fourier analysis of numerical approximations of hyperbolic equations, Philadelphia: Society for Industrial and Applied Mathematics, 7th edition, 1982. 
[45] S. K. Lele, Compact finite-difference schemes with spectral-like resolution, Journal of Computational Physics 103 (1992) 16-42.

[46] C. K. W. Tam, J. C. Webb, Dispersion-relation-preserving finite difference schemes for computational acoustic, Journal of Computational Physics 107 (1993) 262-281.

[47] Z. J. Wang, R. F. Chen, Optimized weighted essentially nonoscillatory schemes for linear waves with discontinuity, Journal of Computational Physics 174 (2001) 381-404.

[48] M. P. Martín, E. M. Taylor, M. Wu, V. G. Weirs, A bandwidthoptimized WENO scheme for effective direct numerical simulation of compressible turbulence, Journal of Computational Physics 220 (2006) 270-289.

[49] G. S. Jiang, C. W. Shu, Efficient implementation of weighted ENO schemes, Journal of Computational Physics 126 (1996) 202-228.

[50] E. M. Taylor, M. Wu, M. P. Martín, Optimization of nonlinear error for weighted essentially non-oscillatory methods in direct numerical simulations of compressible turbulence, Journal of Computational Physics 223 (2007) 384-397.

[51] P. Batten, N. Clarke, C. Lambert, D. M. Causon, On the choice of wave speeds for the HLLC Riemann solver, SIAM Journal on Scientific Computing 18 (1997) 1553-1570.

[52] B. Van Leer, Towards the ultimate conservative difference scheme. v. 
a second-order sequel to Godunov's method, Journal of Computational Physics 32 (1979) 101-136.

[53] J. A. Greenough, W. J. Rider, A quantitative comparison of numerical methods for the compressible euler equations: fifth-order WENO and piecewise-linear Godunov, Journal of Computational Physics 196 (2004) 251-281.

[54] J. W. Jacobs, V. V. Krivet, Experiments on the late-time development of single-mode Richtmyer-Meshkov instability, Physics of Fluids 17 (2005) 034105.

[55] B. D. Collins, J. W. Jacobs, PLIF flow visualization and measurements of the Richtmyer-Meshkov instability of an air $/ \mathrm{SF}_{6}$ interface, Journal of Fluid Mechanics 464 (2002) 113-136.

[56] A. D. Mosedale, D. Drikakis, Assessment of very high order of accuracy in implicit LES models, Journal of Fluids Engineering 129 (2007) 14971503.

[57] D. Drikakis, P. K. Smolarkiewicz, On spurious vortical structures, Journal of Computational Physics 172 (2001) 309-325.

[58] D. Drikakis, M. L. G., P. K. Smolarkiewicz, On 'spurious' eddies, International Journal for Numerical Methods in Fluids 40 (2002) 313-322.

[59] M. Latini, O. Schilling, W. S. Don, Effects of WENO flux reconstruction order and spatial resolution on reshocked two-dimensional RichtmyerMeshkov instability, Journal of Computational Physics 221 (2007) 805836. 
[60] J.-F. Haas, B. Sturtevant, Interaction of weak shock waves with cylindrical and spherical gas inhomogeneities, Journal of Fluid Mechanics 181 (1987) 41-76. 